\title{
Radionuclide Sensors for Water Monitoring
}

(Project Number: 70179)

\section{Principal Investigator}

Jay W. Grate

Pacific Northwest National Laboratory

P.O. Box 999, MSIN K8-93

Richland, WA 99352

(509) 376-4242 (phone)

(509) 376-5106 (fax)

jwgrate@pnl.gov

\section{Co-Investigators}

Oleg B. Egorov

Pacific Northwest National Laboratory

P.O. Box 999, MSIN P7-22

Richland, WA 99352

(509) 376-3485 (phone)

(509) 372-2156 (fax)

oleg.egorov@pnl.gov

Timothy A. DeVol

Clemson University

Clemson, SC 29634-0919

(864) 656-1014 (phone)

tim.devol@ces.clemson.edu

\section{Graduate and Undergraduate Students}

James E. Roane, Ph.D.

Hui Tan, Ph.D.

Lara Drumm, M.S.

Jenny Masters, junior, Chemical Engineering

Tobias Gemm, junior, Environmental Planning

Sunil Bector, junior, Biological Systems Engineering 


\section{Research Objective}

Radionuclide contamination in the soil and groundwater at U.S. Department of Energy (DOE) sites is a severe problem requiring monitoring and remediation. Radionuclide measurement techniques are needed to monitor surface waters, groundwater, and process waters. Typically, water samples are collected and transported to the analytical laboratory where costly radiochemical analyses are performed. To date, there has been very little development of selective radionuclide sensors for alpha- and beta-emitting radionuclides such as ${ }^{90} \mathrm{Sr},{ }^{99} \mathrm{Tc}$, and various actinides of interest.

The objective of this project is to investigate novel sensor concepts and materials for sensitive and selective determination of beta- and alpha-emitting radionuclide contaminants in water. To meet the requirements for low-level, isotope-specific detection, the proposed sensors are based on radiometric detection. As a means to address the fundamental challenge of short ranges of beta and alpha particles in water, our overall approach is based on localization of preconcentration/separation chemistries directly on or within the active area of a radioactivity detector, using automated microfluidics for sample manipulation and sensor regeneration or renewal.

The outcome of these investigations will be the knowledge necessary to choose appropriate chemistries for selective preconcentration of radionuclides from environmental samples, new materials that combine chemical selectivity with scintillating properties, new materials that add chemical selectivity to solid-state diode detectors, new preconcentrating column sensors, and improved instrumentation and signal processing for selective radionuclide sensors. New knowledge will provide the basis for designing effective probes and instrumentation for field analytical chemistry.

\section{Research Progress and Implications}

This report summarizes work through the first 1.5 years of a 3-year program. The initial ongoing effort is directed at the investigation of the preconcentration column sensor concepts, development and characterization of the selective scintillating microspheres (SSMs), and development of the new scintillation detection systems. The preconcentrating minicolumn radionuclide sensor is based on the use of dual-functionality bead materials. These materials are designed to incorporate both selective separation chemistry for analyte preconcentration and localization within the detector, and scintillating properties, so that radioactivity of retained species can be converted to a measurable light output. To date, we have achieved progress in SSM materials prepared by co-immobilization of selective organic extractants and scintillating fluors within inert macroporous polymeric beads, immobilization of selective organic extractants on scintillating glass, and by physically mixing sorbent and scintillating bead materials to yield mixed-bed sensor column. Scintillating sensor materials selective for $\mathrm{Tc}, \mathrm{Sr}$, and actinides were prepared and evaluated. 
Using pertechnetate-selective scintillating beads prepared by co-immobilization of selective extractants and scintillating fluors, we have demonstrated the application of SSM resins for the on-line characterization of ${ }^{99} \mathrm{TcO}_{4}{ }^{-}$(pertechnetate) in acidified groundwater. For on-line measurements, the resin is used in conjunction with an on-line flow-cell scintillation detector and an automated fluid handling system. The results of these tests clearly indicate that pertechnetate can be readily detected in acidified groundwater samples below the U.S. Safe Drinking Water Act maximum concentration level of $900 \mathrm{pCi} / \mathrm{L}$. Nevertheless, long-term material stability and analysis of the unacidified groundwater remain to be problematic when using these sensor materials. To address these challenges, we are evaluating sensor configurations based on a mixed-bed column of anion exchange and polymer scintillator beads. Using mixed-bed sensor columns, we demonstrated the feasibility of ${ }^{99} \mathrm{Tc}$ sensing in unacidified groundwater with detection efficiency of 25\% and excellent long-term sensor column stability. This approach appears especially promising for the development of a ${ }^{99} \mathrm{Tc}$ sensor probe suitable for down-well groundwater monitoring.

Research has commenced on preconcentrating column sensors for monitoring of radiostrontium in aqueous solutions. Strontium-selective dual-functionality sensor materials based on crownether extractant have been realized as 1) a mixed bed of extraction chromatographic resin and granular scintillator, 2) extraction chromatographic material coated on the surface of a scintillating glass, and 3) SSMs prepared by co-immobilization of fluors and extractants. As with the pertechnetate on-line measurements, the resin is used in conjunction with a flow-cell scintillation detection and automated fluid handling system. Degradation of the strontium-SSM with continuous reuse was observed but was not as significant as in the case of ${ }^{99} \mathrm{Tc}$. We have characterized the detection efficiency, minimum detectable concentration, selectivity, loading capacity, capacity factor, and regeneration capability of these dual-functionality resins for ${ }^{89,90} \mathrm{Sr}$ quantification. One of the limitations of the crown ether-based separation chemistry is the need for significant sample acidification. Separation chemistries that work in dilute acid or under groundwater $\mathrm{pHs}$ would be advantageous. We evaluated solid-phase extraction and inorganic sorbent materials for the purpose of Sr capture from groundwater. These two materials were capable of efficient $\mathrm{Sr}$ uptake from groundwater without the need for sample acidification. Mixed-bed column sensors are being evaluated using these sorbent chemistries. Detection efficiencies as high as $50 \%$ were observed, and sensor regeneration was possible.

We have developed and characterized actinide-SSMs to determine feasibility for sequential elution of radioactive species and gross alpha-radiation monitoring. Tests have indicated that sequential separation is feasible using actinide-selective SSM, with results comparing well with standard radiochemical techniques. The absolute detection efficiency of the actinide-SSM depends on alpha energy and ranges from $\sim 80 \%$ to nearly $100 \%$. For gross alpha-radiation monitoring, we have investigated extractive scintillator resins using actinide-selective extractant and actinide-selective chelating resin. Both separation chemistries exhibit strong affinity for tri-, tetra- and hexavalent oxidation state actinides in dilute acids. Scintillation functionality was introduced by 1) producing a mixed-bed of plastic scintillator or scintillating glass beads and extraction chromatographic resin, 2) loading extractant on inorganic scintillator beads, and 
3) introducing scintillation fluors during polymerization and/or by impregnating polymer beads with scintillator fluors. The real-time quantification of natural uranium in groundwater was demonstrated using actinide-selective SSMs.

Development of a flow-cell detection system with digital data acquisition continues. The photomultiplier tube approach to scintillation light detection followed by complete digital signal processing has been demonstrated. The development of a photodiode (PD) approach is nearly complete, but we did not reap the benefit that we had hoped. Because there is no gain associated with the PD, signals from the PDs were amplified and shaped by Canberra 2004 charge-sensitive preamplifier and Princeton Gamma-Tech linear amplifier before being connected to the digital oscilloscope. We achieved pulse shape discrimination of the Parylene $\mathrm{C}$ coated CsI:Tl granular scintillator using the zero-crossing technique. The alpha-particle interactions in the scintillator resulted in a signal that was easily digitized. The beta interactions in the scintillator resulted in a signal that was only slightly above the noise level of the PD, making signal processing problematic. To be able to use the PD system, the PD will need to be cooled to decrease the thermonic noise. Alternatively, an avalanche photodiode or hybrid photomultiplier tube must be used to increase the signal. We are currently evaluating our options to determine if the compact size and reduced power consumption of the PD approach outweighs the decreased signal-tonoise.

During the summer of 2000, Tim DeVol from the Clemson team spent a week with Oleg Egorov at Pacific Northwest National Laboratory. This visit targeted research on chemically modified diode detectors for environmental monitoring of alpha-radiation. We solved some critical problems regarding diode packaging and operation in contact with solution, signal processing, and signal-to-noise. However, further work is necessary regarding the reaction kinetics and optimization of the flow cell for efficient analyte capture on the diode surface.

\section{Planned Activities}

During the remainder of the second and third years, we will direct our activities at further development of SSM materials and sensor configurations for ${ }^{99} \mathrm{Tc},{ }^{90} \mathrm{Sr}$, and actinides as well as fundamental understanding of the radiation interaction and light collection efficiency of the dualfunctionality sensor materials. Development of scintillating materials with reduced leaching of the fluor will continue. We are investigating alternatives to manufactured scintillating macroporous resin. We plan to collaborate with the University of Arkansas, Little Rock to investigate the feasibility of producing dual-functionality composite materials using microencapsulation technology. We are beginning to develop and apply stochastic modeling techniques to better understand the radiation energy deposition in a heterogeneous flow-cell detector as well as the light collection efficiency. We will continue research on chemically selective diode detection for quantification of alpha and beta emitters in solution. 


\section{Information Access}

DeVol TA. 2000. "Chromatographic Separation and Measurement of Charged-Particle Emitting Radionuclides," Eichrom Technologies, Inc. Eastern Users' Group Workshop, Augusta, Georgia (May 16, 2000).

DeVol TA, JM Duffey, and A Paulenova. 2000. "Combination Extraction Chromatography and Scintillation Detection Resin for Quantification of Strontium in Aqueous Solutions," presented at Spectrum 2000, Chattanooga, Tennessee (September 24-28, 2000).

DeVol TA, OB Egorov, JE Roane, A Paulenova, and JW Grate. 2000. "Extractive Scintillating Microspheres for ${ }^{89,90} \mathrm{Sr}$ Quantification in Aqueous Solutions," presented at Gordon Conference on Nuclear Waste and Energy, New London, New Hampshire (July 16-21, 2000).

DeVol TA, JE Roane, JM Williamson, JM Duffey, and JT Harvey. 2000. "Development of Scintillating Extraction Media for Separation and Measurement of Charged-Particle Emitting Radionuclides in Aqueous Solutions," Radioactivity and Radiochemistry, 11(1):34-46).

DeVol TA, JM Duffey, and A Paulenova. 2001. "Combined Extraction Chromatography and Scintillation Detection for On-line and Off-line Monitoring of Strontium in Aqueous Solutions," presented at the Fifth International Conference on Methods and Application of Radioanalytical Chemistry, Kailua-Kona, Hawaii, April 9-14, 2000, and accepted for publication in Journal of Radioanalytical and Nuclear Chemistry, Vol. 249, No. 2.

DeVol TA, OB Egorov, JE Roane, A Paulenova, and JW Grate. 2001. "Extractive Scintillating Microspheres for ${ }^{99}$ Tc Quantification in Aqueous Solutions," presented at the Fifth International Conference on Methods and Application of Radioanalytical Chemistry, Kailua-Kona, Hawaii, April 9-14, 2000, and accepted for publication in Journal of Radioanalytical and Nuclear Chemistry, Vol. 249, No. 1.

DeVol TA, JE Roane, and JD Leyba. 2001. "Application of Extractive Scintillator Resins to Quantification of Beta-Emitting Radionuclides in Aqueous Solutions," presented at LCS 2001, Karlsruhe, Germany (May 7-11, 2001) and submitted to Radiocarbon for publication.

Drumm L and TA DeVol. 2001. "Gross Alpha Radiation Monitoring in Natural Waters," presented at LCS 2001, Karlsruhe, Germany (May 7-11, 2001).

Egorov OB, SK Fiskum, MJ O’Hara, and JW Grate. 1999. “Radionuclide Sensors Based on Chemically Selective Scintillating Microspheres: Renewable Column Sensor for Analysis of ${ }^{99}$ Tc in Water," Anal. Chem. 71, 5420-5429.

Egorov OB and JW Grate. 2000. "Automating Analytical Separations in Radiochemistry," presented at the Gordon Conference on Nuclear Waste and Energy, New London, New Hampshire, July 16-21. 
Egorov OB and JW Grate. 2000. "Automated Radionuclide Separations, Analysis and Sensing," presented at the 46th Annual Conference on Bioassay, Analytical, and Environmental Radiochemistry, November 12-17, 2000, Seattle, Washington.

Roane JE and TA DeVol. 2001. "Scintillating Extraction Chromatography Coupled with Pulse Height Spectrum Analysis for Actinide Separation and Analysis," presented at the 2001 American Radiation Safety Conference \& Exposition, Cleveland, Ohio (June 10-14, 2001).

Tan H and TA DeVol. 2000. "Development of a Digital Alpha/Beta Pulse Shape Discriminating System Utilizing CsI(Tl)/Photodiode," presented at the 44th annual meeting of the Health Physics Society, Denver, Colorado (June 24-29, 2000).

Tan H, RA Fjeld, and TA DeVol. 2000. "Digital Alpha/Beta Pulse Shape Discrimination of CsI:Tl for On-Line Measurement of Aqueous Radioactivity," IEEE Trans. Nuc. Sci., 47(4):1516-1521. 